\title{
ON THE ROBUST STABILITY OF NEUTRAL SYSTEMS WITH TIME-VARYING DELAYS
}

\author{
V. J. S. Leite ${ }^{*}$ P. L. D. Peres ${ }^{* *}$ E. B. Castelan ${ }^{* * *}$ \\ S. Tarbouriech ${ }^{* * * *}$ \\ * UnED Divinópolis - CEFET-MG - R. Monte Santo, 319 \\ 35502-036, Divinópolis - MG - Brazil \\ E-mail: valter@div.cefetmg.br \\ ** DT/FEEC - University of Campinas \\ CP 6101, 13081-970 Campinas - SP - Brazil \\ E-mail: peres@dt.fee.unicamp.br \\ *** DAS - CTC - Federal University of Santa Catarina \\ 88040-900, Florianópolis - SC - Brazil \\ E-mail: eugenio@das.ufsc.br

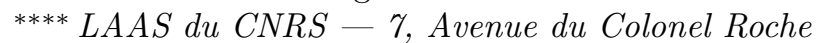 \\ 31077 Toulouse CEDEX 4 - France \\ E-mail: tarbour@laas.fr
}

\begin{abstract}
Linear matrix inequality conditions are given for the robust stability of neutral systems with time-varying delays in the state vector. All the system matrices are supposed to be time-invariant, belonging to a known polytope. The robust stability is assured by means of a parameter-dependent LyapunovKrasovskii functional, independently of the size of the time-varying delays. A numerical example illustrates the results. Copyright (C)IFAC 2005.
\end{abstract}

Keywords: Time-delay systems, neutral systems, robust stability, parameter-dependent quadratic functions, linear matrix inequalities

\section{INTRODUCTION}

Time-delays occur very frequently in many dynamic systems and can be of crucial importance for the system stability (see Dugard and Verriest (1997), Hale and Lunel (1993), Kolmanovskii and Richard (1999), Malek-Zavarei and Jamshidi (1987) and references therein). The analysis of stability becomes more complicated in the presence of uncertain parameters, and many works have been published during the last decades providing sufficient conditions for the robust stability of time-delay systems. The Lyapunov-Krasovskii

\footnotetext{
1 This work is partially supported by the Brazilian agencies CAPES, CNPq and FAPEMIG.
}

functionals are undoubtedly one of the main tools to derive stability conditions for this kind of systems (Kolmanovskii et al., 1999), (Skorodinskii, 1990) and there have been efforts to characterize the robust stability by means of linear matrix inequalities (LMIs), which can be solved in polynomial time through interior point algorithms (Boyd et al., 1994), (Gahinet et al., 1995). Since the delay-dependent conditions for robust stability of retarded systems are in general conservative or, when more accurate, demand a high computation level, it is of great interest to obtain less conservative LMI based delay-independent conditions for uncertain retarded systems. 
The main focus of this paper is on the robust stability of neutral systems (i.e. systems whose dynamics depends also on the time-derivative of past states). Transmission lines or dynamical processes including steam or water pipes that can be described by linear hyperbolic differential equations with mixed initial and derivative boundary conditions are examples of neutral systems (Hale and Lunel, 1993), (Niculescu, 2001), (Bellen et al., 1999). Among several papers dealing with this class of systems, on may cite Niculescu (2001) (both delay-dependent and delay-independent conditions, time-invariant delays and no uncertainty), Bin et al. (2003) (delay-dependent conditions, time-invariant delays and no uncertainty), Verriest (1999) (delayindependent Riccati based conditions for timevarying systems and time-varying delays), $\mathrm{Xu}$ et al. (2003) (norm-bounded uncertain systems, time-invariant delays), Bliman (2002) (delay-independent sufficient LMI conditions tending to necessity as the complexity increases, precisely known $E$ matrix, polytopic uncertainties and time-invariant delays), Fridman (2001) (no uncertainty, introduces a descriptor system approach). Other relevant results include Park (2003), Cao and He (2004), Chen (2003), Park et al. (2004), Chen (2004), Fu (2004), Park and Won (2000), Park (2001), Han (2002), Han (2004), Ivănescu et al. (2003). Most of these works consider only time-invariant delays and, as a general rule, they consider that the same delay affects both the delayed states and the time-derivative of the delayed states.

To deal with the presence of uncertainty, the quadratic stability approach (Barmish, 1985) is used in most of cases. In the context of robust stability of uncertain linear systems, improved conditions have been obtained through parameterdependent Lyapunov functions (de Oliveira et al., 1999), (Leite and Peres, 2003), (Peaucelle et al., 2000), (Ramos and Peres, 2001), (Ramos and Peres, 2002). This paper extends these recent results to the robust stability analysis of uncertain neutral systems with time-varying delays. On the contrary of the majority of papers dealing with this subject, different time-delays are considered in the delayed states and in the time-derivative of the delayed states. No model transformation (which introduces new dynamics, as discussed in Gu and Niculescu (2001)) is used and all the system matrices are supposed to belong to a polytope with known vertices. Sufficient LMI conditions are given for the existence of a parameter dependent Lyapunov-Krasovskii functional assuring the robust stability of the uncertain system irrespective of the time-varying time-delays. Although presented for the case of single delays, the conditions can easily be extended to the case of multiple delays. The formulation presented encompasses previous results from the literature, such as robust stability of uncertain systems without delays or robust stability of uncertain retarded systems as special cases. A numerical example illustrates the results.

\subsection{Notation}

The notation used in this paper is quite standard. $\mathbb{R}^{+}$is the set of nonnegative real numbers. I and $\mathbf{0}$ denote, respectively, the identity matrix and the null matrix of appropriate dimensions. $M>\mathbf{0}(<\mathbf{0})$ means that matrix $M$ is positive (negative) definite. $\mathcal{C}_{\tau}=\mathcal{C}\left([-\tau, 0], \mathbb{R}^{n}\right)$ denotes the Banach space of continuous vector functions mapping the interval $[-\tau, 0]$ into $\mathbb{R}^{n}$ with the topology of uniform convergence. $\mathcal{C}_{\tau}^{v}$ is the set defined by $\mathcal{C}_{\tau}^{v}=\left\{\phi \in \mathcal{C}_{\tau} ;\|\phi\|_{c}<v, v>0\right\}$. The symbol $\star$ stands for symmetric blocks in the LMIs.

\section{PRELIMINARIES}

Consider the following class of uncertain neutral linear system

$$
\begin{gathered}
\left.\frac{\partial}{\partial t} \mathcal{D}\left(x_{t}\right)=A(\alpha) x(t)+A_{\theta}(\alpha) x(t-\theta(t))\right) \\
\mathcal{D}\left(x_{t}\right) \triangleq x(t)-E(\alpha) x(t-\tau(t))
\end{gathered}
$$

with the initial condition

$$
\begin{gathered}
x\left(t_{0}+\xi\right)=\phi(\xi), \forall \xi \in[-\varsigma, 0], \quad\left(t_{0}, \phi\right) \in \mathbb{R}^{+} \times \mathcal{C}_{\varsigma}^{v} \\
\varsigma \triangleq \max \{\theta(t), \tau(t))\}, \theta(t) \geq 0, \tau(t) \geq 0, \forall t \quad(4)
\end{gathered}
$$

where $x(t) \in \mathbb{R}^{n}$ is the state and $\theta(t) \in \mathbb{R}^{+}$and $\tau(t) \in \mathbb{R}^{+}$are time-varying delays. The timeinvariant system matrices $E(\alpha), A(\alpha)$ and $A_{\theta}(\alpha)$ are not precisely known, but belong to a polytopic domain $\mathcal{P}$ with known vertices $E_{j}, A_{j}, A_{\theta j}$ (or $\left.\left(E, A, A_{\theta}\right)_{j}\right)$ given by

$$
\begin{aligned}
\mathcal{P}=\left\{\left(E, A, A_{\theta}\right)(\alpha)\right. & \in \mathbb{R}^{n \times 3 n}: \\
\left(E, A, A_{\theta}\right)(\alpha)= & \sum_{j=1}^{N} \alpha_{j}\left(E, A, A_{\theta}\right)_{j} ; \\
& \left.\sum_{j=1}^{N} \alpha_{j}=1 ; \alpha_{j} \geq 0\right\}
\end{aligned}
$$

Any matrix triple $\left(E, A, A_{\theta}\right)(\alpha)$ inside $\mathcal{P}$ can be written as a convex combination of the vertices $\left(E, A, A_{\theta}\right)_{j}$ of the uncertainty polytope in terms of $\alpha, \alpha_{j} \geq 0, \sum_{j=1}^{N} \alpha_{j}=1$.

This paper addresses the following problem:

Problem 1. Determine, if possible, conditions assuring that the uncertain neutral linear system (1)-(5) is robustly stable independently from the time-delay values $\theta(t)$ and $\tau(t)$. 
Sufficient conditions for solving Problem 1 are given in terms of LMIs in the sequel. A parameter dependent Lyapunov-Krasovskii functional is used, providing delay-independent convex conditions which are less conservative results than similar results obtained with the quadratic approach (constant matrices in the Lyapunov-Krasovskii functional).

\section{ROBUST DELAY-INDEPENDENT STABILITY}

First, a general matrix inequality condition (with infinite dimension on $\alpha$ ) is given. From this condition, a convex one with finite number of LMIs is obtained by choosing some matrix variables as independent of the uncertain parameter $\alpha$.

The following lemma, from stability theory of Lyapunov, is used in the sequel.

Lemma 1. The uncertain neutral system (1)-(5) is robustly stable independently from the timevarying delays $\theta(t)$ and $\tau(t)$ if there exist symmetric positive definite matrices $P(\alpha) \in \mathbb{R}^{n \times n}$, $S_{1}(\alpha) \in \mathbb{R}^{n \times n}$ and $S_{2}(\alpha) \in \mathbb{R}^{n \times n}$ such that

$$
\begin{gathered}
V(\alpha, x(t), \theta(t), \tau(t))=\mathcal{D}\left(x_{t}\right)^{\prime} P(\alpha) \mathcal{D}\left(x_{t}\right) \\
+\int_{t-\theta(t)}^{t} x(\sigma)^{\prime} S_{1}(\alpha) x(\sigma) d \sigma \\
+\int_{t-\tau(t)}^{t} x(\varepsilon)^{\prime} S_{2}(\alpha) x(\varepsilon) d \varepsilon>0 \\
\forall\left(x(t)^{\prime}, x(t-\theta(t))^{\prime}, x\left(t-\tau(t)^{\prime}\right)^{\prime} \neq \mathbf{0}\right. \text { and } \\
\frac{\partial}{\partial t} V(\alpha, x(t), \theta(t), \tau(t))<0
\end{gathered}
$$

An important remark about Lemma 1 is that it does not provide a method to obtain matrices $P(\alpha), S_{1}(\alpha)$ and $S_{2}(\alpha)$. Note also that no bounds on the rate of variation of the time-delays are imposed. In order to provide sufficient conditions to Lemma 1, thus solving Problem 1, the following bounds are assumed:

$$
\begin{aligned}
|\dot{\theta}(t)| \leq \bar{\theta}<1 \\
|\dot{\tau}(t)| \leq \bar{\tau}<1
\end{aligned}
$$

Define also the scalars

$$
\beta_{\bar{\theta}} \triangleq(1-\bar{\theta}) ; \beta_{\bar{\tau}} \triangleq(1-\bar{\tau})
$$

Theorem 1. The following equivalent conditions guarantee the delay-independent robust stability of the neutral system (1)-(5) with time-varying delays with bounded variation rates given in (8)(9) and are sufficient to verify Lemma 1, thus assuring a solution to Problem 1: i) There exist symmetric positive definite matrices $P(\alpha) \in \mathbb{R}^{n \times n}, S_{1}(\alpha) \in \mathbb{R}^{n \times n}, S_{2}(\alpha) \in \mathbb{R}^{n \times n}$ such that

$$
\begin{aligned}
& \Theta(\alpha) \triangleq \\
& {\left[\begin{array}{ccc}
\Psi & P(\alpha) A_{\theta}(\alpha) & -A(\alpha)^{\prime} P(\alpha) E(\alpha) \\
\star & -\beta_{\bar{\theta}} S_{1}(\alpha) & -A_{\theta}(\alpha)^{\prime} P(\alpha) E(\alpha) \\
\star & \star & -\beta_{\bar{\tau}} S_{2}(\alpha)
\end{array}\right]<\mathbf{0}}
\end{aligned}
$$

with

$$
\Psi=A(\alpha)^{\prime} P(\alpha)+P(\alpha) A(\alpha)+S_{1}(\alpha)+S_{2}(\alpha)
$$

ii) There exist symmetric positive definite matrices $P(\alpha) \in \mathbb{R}^{n \times n}, S_{1}(\alpha) \in \mathbb{R}^{n \times n}, S_{2}(\alpha) \in \mathbb{R}^{n \times n}$ and matrices $F_{1}(\alpha), F_{2}(\alpha), G_{1}(\alpha), G_{2}(\alpha), H_{1}(\alpha)$, $H_{2}(\alpha), M_{1}(\alpha), M_{2}(\alpha), N_{1}(\alpha)$ and $N_{2}(\alpha)$ belonging to $\mathbb{R}^{n \times n}$ such that equation (13) holds.

Proof: The fact that $i$ ) is a sufficient condition to solve Problem 1 follows directly from Lemma 1. By evaluating the time-derivative of $V(\cdot)$, with $\mathcal{D}\left(x_{t}\right)$ given by $(2)$, one gets $\chi(t)^{\prime} \Theta(\alpha, t) \chi(t)$ with $\chi(t) \equiv\left[x(t)^{\prime} x(t-\theta(t))^{\prime} x(t-\tau(t))^{\prime}\right]^{\prime}$ and

$$
\begin{aligned}
\tilde{\Theta}(\alpha, t) & \triangleq \\
& {\left[\begin{array}{ccc}
\Psi & P(\alpha) A_{\theta}(\alpha) & -A(\alpha)^{\prime} P(\alpha) E(\alpha) \\
\star & -\beta_{\dot{\theta}} S_{1}(\alpha) & -A_{\theta}(\alpha)^{\prime} P(\alpha) E(\alpha) \\
\star & \star & -\beta_{\dot{\tau}} S_{2}(\alpha)
\end{array}\right] }
\end{aligned}
$$

where $\beta_{\dot{\theta}} \triangleq(1-\dot{\theta}(t)), \beta_{\dot{\tau}} \triangleq(1-\dot{\tau}(t))$ and $\Psi$ is given in (12). Now, using (8)-(9) and (11) one has $\tilde{\Theta}(\alpha, t) \leq \Theta(\alpha)<\mathbf{0}$ thus assuring the conditions of Lemma 1. The fact that ii) $\Rightarrow$ i) can be obtained by applying the congruence transformation $\Theta(\alpha) \equiv \mathcal{U}^{\prime} \Xi(\alpha) \mathcal{U}$ with

$$
\mathcal{U} \triangleq\left[\begin{array}{ccccc}
A(\alpha)^{\prime} & \mathbf{I} & \mathbf{I} & \mathbf{0} & \mathbf{0} \\
A_{\theta}(\alpha)^{\prime} & \mathbf{0} & \mathbf{0} & \mathbf{I} & \mathbf{0} \\
\mathbf{0} & -E(\alpha)^{\prime} & \mathbf{0} & \mathbf{0} & \mathbf{I}
\end{array}\right]^{\prime}
$$

and the converse can be proved by using the Finsler's Lemma (see for instance de Oliveira and Skelton (2001)).

The conditions presented in Theorem 1 do not depend on $\tau(t)$ and $\theta(t)$, but only on their maximum rates of variation, respectively $\bar{\tau}$ and $\bar{\theta}$. Moreover, Theorem 1 could be used to verify the robust stability of a neutral system belonging to any uncertainty domain parametrized on $\alpha$, at the price of testing all possible values of $\alpha$. A numerically efficient test with a finite number of LMIs assuring the conditions of Lemma 1 can be obtained by imposing the following structure on matrices $P(\alpha), S_{1}(\alpha), S_{2}(\alpha)$

$$
\begin{aligned}
& P(\alpha)=\sum_{j=1}^{N} \alpha_{j} P_{j} ; S_{1}(\alpha)=\sum_{j=1}^{N} \alpha_{j} S_{1 j} ; \\
& S_{2}(\alpha)=\sum_{j=1}^{N} \alpha_{j} S_{2 j} ; \quad \sum_{j=1}^{N} \alpha_{j}=1, \alpha_{j} \geq 0
\end{aligned}
$$




$$
\Xi(\alpha) \triangleq\left[\begin{array}{cc}
F_{1}(\alpha)+F_{1}(\alpha) & P(\alpha)+F_{2}(\alpha)+G_{1}(\alpha)^{\prime} \\
\star & G_{2}(\alpha)+G_{2}(\alpha)^{\prime} \\
& \star \\
\star & \star \\
\star & \star \\
\star & M_{1}(\alpha)^{\prime}-F_{1}(\alpha) A_{\theta}(\alpha) \\
& M_{2}(\alpha)^{\prime}-G_{1}(\alpha) A_{\theta}(\alpha) \\
& -\left(H_{1}(\alpha) A_{\theta}(\alpha)\right. \\
& \left.+A(\alpha)^{\prime} M_{1}(\alpha)^{\prime}+M_{2}(\alpha)^{\prime}\right) \\
& -\beta_{\bar{\theta}} S_{1}(\alpha)+M_{1}(\alpha) A_{\theta}(\alpha) \\
& +A_{\theta}(\alpha)^{\prime} M_{1}(\alpha)^{\prime} \\
& \star
\end{array}\right.
$$

and by choosing $F_{1}(\alpha)=F_{1}, F_{2}(\alpha)=F_{2}$, $G_{1}(\alpha)=G_{1}, G_{2}(\alpha)=G_{2}, H_{1}(\alpha)=H_{1}, H_{2}(\alpha)=$ $H_{2}, M_{1}(\alpha)=M_{1}, M_{2}(\alpha)=M_{2}, N_{1}(\alpha)=N_{1}$ and $N_{2}(\alpha)=N_{2}$.

Theorem 2. If there exist symmetric positive definite matrices $P_{j} \in \mathbb{R}^{n \times n}, S_{1 j} \in \mathbb{R}^{n \times n}, S_{2 j} \in$ $\mathbb{R}^{n \times n}, j=1, \ldots, N$ and matrices $F_{1}, F_{2}, G_{1}, G_{2}$, $H_{1}, H_{2}, M_{1}, M_{2}, N_{1}$ and $N_{2}$ belonging to $\mathbb{R}^{n \times n}$ such that

$$
\Xi_{j} \triangleq\left[\begin{array}{ccc}
F_{1}+F_{1} & P_{j}+F_{2}+G_{1}^{\prime} & H_{1}^{\prime}-\left(F_{1} A_{j}+F_{2}\right) \\
\star & G_{2}+G_{2}^{\prime} & H_{2}^{\prime}-\left(G_{1} A_{j}+G_{2}\right) \\
& & S_{1 j}+S_{2 j}-\left(H_{1} A_{j}\right. \\
\star & \star & \left.+A_{j}^{\prime} H_{1}^{\prime}+H_{2}+H_{2}^{\prime}\right) \\
\star & \star & \star \\
\star & \star & \star
\end{array}\right.
$$

$$
\left.\begin{array}{cc}
M_{1}^{\prime}-F_{1} A_{\theta j} & N_{1}^{\prime}+F_{2} E_{j} \\
M_{2}^{\prime}-G_{1} A_{\theta j} & N_{2}^{\prime}+G_{2} E_{j} \\
-\left(H_{1} A_{\theta j}\right. & H_{2} E_{j} \\
\left.+A_{j}^{\prime} M_{1}^{\prime}+M_{2}^{\prime}\right) & -\left(A_{j}^{\prime} N_{1}^{\prime}+N_{2}^{\prime}\right) \\
-\beta_{\bar{\theta}} S_{1 j}+M_{1} A_{\theta j} & M_{2} E_{j}-A_{\theta j}^{\prime} N_{1}^{\prime} \\
+A_{\theta j}^{\prime} M_{1}^{\prime} & -\beta_{\bar{\tau}} S_{2 j} \\
\star & +N_{2} E_{j}+E_{j}^{\prime} N_{2}^{\prime}
\end{array}\right]<\mathbf{0}
$$

then, Lemma 1 holds with $P(\alpha), S_{1}(\alpha)$ and $S_{2}(\alpha)$ given by (14) thus assuring a solution to Problem 1.

Proof: Clearly, $P(\alpha), S_{1}(\alpha)$ and $S_{2}(\alpha)$ given by (14) with $P_{j}=P_{j}^{\prime}>0, S_{1 j}=S_{1 j}^{\prime}>0, S_{2 j}=$ $S_{2 j}^{\prime}>0, j=1, \ldots, N$ are parameter dependent positive definite matrices. Multiplying (15) by $\alpha_{j}, \alpha_{j} \geq 0, \sum_{j=1}^{N} \alpha_{j}=1$ and summing up on $j=1, \ldots, N(13)$ is verified.

Robust stability conditions for special cases of system (1)-(5) can be obtained directly from Theorem 2 (as well as from Theorem 1). These delayindependent robust stability conditions can be

$$
\left.\begin{array}{c}
H_{1}(\alpha)^{\prime}-\left(F_{1}(\alpha) A(\alpha)+F_{2}(\alpha)\right) \\
H_{2}(\alpha)^{\prime}-\left(G_{1}(\alpha) A(\alpha)+G_{2}(\alpha)\right) \\
S_{1}(\alpha)+S_{2}(\alpha)-\left(H_{1}(\alpha) A(\alpha)\right. \\
\left.+A(\alpha)^{\prime} H_{1}(\alpha)^{\prime}+H_{2}(\alpha)+H_{2}(\alpha)^{\prime}\right) \\
\star \\
\star \\
N_{1}(\alpha)^{\prime}+F_{2}(\alpha) E(\alpha) \\
N_{2}(\alpha)^{\prime}+G_{2}(\alpha) E(\alpha) \\
H_{2}(\alpha) E(\alpha)-\left(A(\alpha)^{\prime} N_{1}(\alpha)^{\prime}+N_{2}(\alpha)^{\prime}\right) \\
M_{2}(\alpha) E(\alpha)-A_{\theta}(\alpha)^{\prime} N_{1}(\alpha)^{\prime} \\
-\beta_{\bar{\tau}} S_{2}(\alpha)+N_{2}(\alpha) E(\alpha)+E(\alpha)^{\prime} N_{2}(\alpha)^{\prime}
\end{array}\right]<\mathbf{0}
$$

obtained by changing the Lyapunov-Krasovskii functional and applying appropriate congruence transformations in the LMIs of (15).

For instance, consider $E(\alpha) \equiv \mathbf{0}$ in (1), implying that the neutral characteristic of the system is lost. The system is now a standard retarded uncertain system given by

$$
\dot{x}(t)=A(\alpha) x(t)+A_{\theta}(\alpha) x(t-\theta(t))
$$

By fixing $S_{2}(\alpha) \equiv \mathbf{0}$ in the functional (6), a robust stability condition for system (16) can be obtained from (15) by using the congruence transformation

$$
\Xi_{E j}=T_{E} \Xi_{j} T_{E}^{\prime} ; \quad j=1, \ldots, N
$$

with

$$
T_{E}=\left[\begin{array}{lllll}
\mathbf{I} & 0 & 0 & 0 & 0 \\
0 & \mathbf{I} & \mathbf{I} & 0 & 0 \\
\mathbf{0} & 0 & 0 & \mathbf{I} & 0
\end{array}\right]
$$

as presented in the following corollary.

Corollary 1. If there exist symmetric definite positive matrices $S_{1 j} \in \mathbb{R}^{n \times n}$ and $P_{j} \in \mathbb{R}^{n \times n}$, $j=1, \ldots, N$, matrices $F \in \mathbb{R}^{n \times n}, G \in \mathbb{R}^{n \times n}$, $M \in \mathbb{R}^{n \times n}$ such that

$$
\begin{aligned}
\Xi_{E j} \triangleq & {\left[\begin{array}{cc}
F+F^{\prime} & P_{j}+G^{\prime}-F A_{j} \\
\star & S_{1 j}-G A_{j}-A_{j}^{\prime} G^{\prime} \\
\star & \star \\
M^{\prime}-F A_{\theta j} \\
-G A_{\theta j}-A_{j}^{\prime} M^{\prime} \\
-\beta_{\bar{\theta}} S_{1 j}-M A_{\theta j}-A_{\theta j}^{\prime} M^{\prime}
\end{array}\right]<\mathbf{0} } \\
& j=1, \ldots, N
\end{aligned}
$$

then, the uncertain linear retarded system (16) with time-varying delay $\theta(t)$ subjected to (8) is robustly stable independently from the timedelay.

The variables of Corollary 1 are related to the ones of Theorem 2 in the following way: $F=F_{1}$; $G=G_{1}+H_{1}$ and $M=M_{1}\left(P(\alpha)\right.$ and $S_{1}(\alpha)$ are given by (14) in both cases).

A second case to be investigated is the uncertain neutral system (1) with $A_{\theta}(\alpha) \equiv \mathbf{0}$. The robust 
delay-independent stability condition for this case can be obtained from (15) through the congruence transformation $T_{A_{\theta}} \Xi_{j} T_{A_{\theta}}^{\prime} ; j=1, \ldots, N$ with

$$
T_{A_{\theta}}=\left[\begin{array}{lllll}
\mathbf{I} & 0 & 0 & 0 & 0 \\
\mathbf{0} & \mathbf{I} & 0 & 0 & 0 \\
\mathbf{0} & \mathbf{0} & \mathbf{I} & \mathbf{0} & \mathbf{0} \\
\mathbf{0} & \mathbf{0} & 0 & \mathbf{0} & \mathbf{I}
\end{array}\right]
$$

Finally, consider system (1) without delay, that is, $E(\alpha) \equiv A_{\theta}(\alpha) \equiv \mathbf{0}$ yielding the uncertain continuous-time system $\dot{x}(t)=A(\alpha) x(t)$. The robust stability condition can be obtained by imposing (without loss of generality) $S_{1}(\alpha) \equiv$ $S_{2}(\alpha) \equiv \mathbf{0}$ in functional (6), resulting in the robust stability condition for continuous-time uncertain systems presented in Peaucelle et al. (2000). This can be obtained from (15) by using the congruence transformation $T_{E A_{\theta}} \Xi_{j} T_{E A_{\theta}}^{\prime} ; j=1, \ldots, N$ with

$$
T_{E A_{\theta}}=\left[\begin{array}{lllll}
\mathbf{0} & \mathbf{I} & \mathbf{I} & \mathbf{0} & \mathbf{0} \\
\mathbf{I} & \mathbf{0} & \mathbf{0} & \mathbf{0} & \mathbf{0}
\end{array}\right]
$$

Similar transformations could be applied on condition ii) of Theorem 1.

It is important to emphasize that all the results presented here encompass, as a special case, the quadratic stability based conditions which use constant matrices in the Lyapunov-Krasovskii functional. Those conditions can be obtained by choosing $P(\alpha)=P_{j}=P, S_{1}(\alpha)=S_{1 j}=S_{1}$ and $S_{2}(\alpha)=S_{2 j}=S_{2}$. The numerical complexity of the presented conditions are now given: Theorem 2 and Corollary 1 involve $\mathcal{K}_{T 2}=3 N n(n+$ 1) $/ 2+10 n^{2}$ and $\mathcal{K}_{C 1}=N n(n+1)+3 n^{2}$ scalar variables, respectively, and $\mathcal{L}_{T 2}=5 n$ and $\mathcal{L}_{C 1}=3 n$ LMI rows, being solved in polynomial time by specialized algorithms, as Gahinet et al. (1995), with complexity proportional to $\mathcal{K}^{3} \mathcal{L}$ (other LMI solvers may perform differently).

\section{NUMERICAL EXAMPLE}

Consider the uncertain neutral system with two states, represented in a polytopic domain by three vertices $V_{j} \equiv\left(E, A, A_{\theta}\right)_{j}, j=1,2,3$, given by

$V_{1}=\left[\begin{array}{cc|cc|cc}-0.10 & 0 & -0.7085 & -0.0758 & -0.3543 & -0.0379 \\ 0 & -0.10 & -0.2511 & -0.0269 & -0.1256 & -0.0135\end{array}\right]$

$V_{2}=\left[\begin{array}{cc|cc|cc}-0.15 & 0 & -0.3260 & 0.2616 & -0.1630 & 0.1308 \\ 0 & -0.15 & 0.2980 & -0.2393 & 0.1490 & -0.1196\end{array}\right]$

$V_{3}=\left[\begin{array}{cc|cc|cc}-0.20 & 0 & -0.3957 & 0.3236 & -0.1978 & 0.1618 \\ 0 & -0.20 & 0.3513 & -0.2874 & 0.1756 & -0.1437\end{array}\right]$

Using the results of Theorem 2, it is possible to characterize a region $(\bar{\theta}, \bar{\tau})$ where this system is robustly stable independently of delay values $\theta(t)$ and $\tau(t)$, as shown in Figure 1 (a grid of 0.02 on $\bar{\tau}$ and $\bar{\theta}$ has been used. It is worth to mention that this system cannot be characterized as robustly stable by quadratic stability conditions.

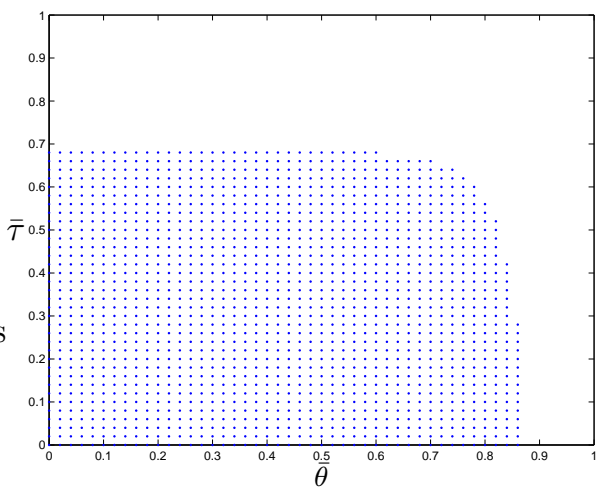

Fig. 1. $(\bar{\theta}, \bar{\tau})$ region for robust delay-independent stability of neutral system (18)-(20) obtained through the conditions of Theorem 2.

\section{CONCLUSION}

Sufficient LMI conditions for the analysis of robust stability of neutral systems with time-varying delays belonging to polytopic domains have been given, based on parameter-dependent LyapunovKrasovskii functionals. Stability conditions for special cases of the neutral system can be obtained from the conditions, which also encompass quadratic stability based results.

\section{REFERENCES}

Barmish, B. R. (1985). Necessary and sufficient conditions for quadratic stabilizability of an uncertain system. Journal of Optimization Theory and Applications 46, 399-408.

Bellen, A., N. Guglielmi and A. E. Ruehli (1999). Methods for linear systems of circuit delay differential equations of neutral type. IEEE Transactions on Circuits and Systems Part I: Fundamental Theory and Applications 46(1), 212-216.

Bin, Y., Z. Ruijun and L. Tao (2003). Delaydependent stability criterion for a class of neutral time-delay systems. In: Proceedings of the 2003 American Control Conference. Denver, CO. pp. 2694-2696.

Bliman, P.-A. (2002). Lyapunov equation for the stability of linear delay systems of retarded and neutral type. IEEE Transactions on Automatic Control 47(2), 327-335.

Boyd, S., L. El Ghaoui, E. Feron and V. Balakrishnan (1994). Linear Matrix Inequalities in System and Control Theory. SIAM Studies in Applied Mathematics. Philadelphia, PA.

Cao, D. Q. and P. He (2004). Stability criteria of linear neutral systems with a single delay. Applied Mathematics and Computation 148(1), 135-143. 
Chen, J.-D. (2003). New stability criteria for a class of neutral systems with discrete and distributed time-delays: an LMI approach. Applied Mathematics and Computation 150(3), 719-736.

Chen, J.-D. (2004). Robust control for uncertain neutral systems with time-delays in state and control input via LMI and GAs. Applied Mathematics and Computation 157(2), 535548.

de Oliveira, M. C. and R. E. Skelton (2001). Stability tests for constrained linear systems. In: Perspectives in Robust Control (S. O. Reza Moheimani, Ed.). Vol. 268 of Lecture Notes in Control and Information Science. pp. 241257. Springer-Verlag. New York.

de Oliveira, M. C., J. Bernussou and J. C. Geromel (1999). A new discrete-time robust stability condition. Systems \& Control Letters 37(4), 261-265.

Dugard, L. and E. I. Verriest (1997). Stability and Control of Time-delay Systems. SpringerVerlag. Berlin, Germany.

Fridman, E. (2001). New Lyapunov-Krasovskii functionals for stability of linear retarded and neutral type systems. Systems \& Control Letters 43(4), 309-319.

$\mathrm{Fu}$, X. (2004). Controllability of abstract neutral functional differential systems with unbounded delay. Applied Mathematics and Computation 151(2), 299-314.

Gahinet, P., A. Nemirovski, A. J. Laub and M. Chilali (1995). LMI Control Toolbox User's Guide. The Math Works Inc., Natick, MA.

Gu, K. and S.-I. Niculescu (2001). Further remarks on additional dynamics in various model transformations of linear delay systems. IEEE Transactions on Automatic Control 46(3), 497-500.

Hale, J. and S. M. V. Lunel (1993). Introduction to Functional Differential Equations. SpringerVerlag. New York.

Han, Q.-L. (2002). Robust stability of uncertain delay-differential systems of neutral type. $A u$ tomatica 38(4), 719-723.

Han, Q.-L. (2004). A descriptor system approach to robust stability of uncertain neutral system with discrete and distributed delays. $A u-$ tomatica 40(10), 1791-1796.

Ivănescu, D., S.-I. Niculescu, L. Dugard, J.-M. Dion and E. I. Verriest (2003). On delaydependent stability for linear neutral systems. Automatica 39(2), 255-261.

Kolmanovskii, V. B. and J. P. Richard (1999). Stability of some linear systems with delays. IEEE Transactions on Automatic Control 44(5), 984-989.

Kolmanovskii, V. B., S. I. Niculescu and J. P. Richard (1999). On the Liapunov-Krasovskii functionals for stability analysis of linear delay systems. International Journal of Control 72(4), 374-384.

Leite, V. J. S. and P. L. D. Peres (2003). An improved LMI condition for robust $\mathcal{D}$-stability of uncertain polytopic systems. IEEE Transactions on Automatic Control 48(3), 500-504.

Malek-Zavarei, M. and M. Jamshidi (1987). Time-Delay Systems: Analysis, Optimization and Applications. North-Holland. Amsterdam, The Netherlands.

Niculescu, S.-I. (2001). On delay-dependent stability under model transformations of some neutral linear systems. International Journal of Control 74(6), 609-617.

Park, J.-H. (2001). A new delay-dependent criterion for neutral systems with multiple delays. Journal of Computational and Applied Mathematics 136(1-2), 177-184.

Park, J. H. (2003). Simple criterion fot asymptotic stability of interval neutral delaydifferential systems. Applied Mathematics Letters 16(7), 1063-1068.

Park, J. H. and S. Won (2000). Stability analysis for neutral delay-differential systems. Journal of The Franklin Institute 337(1), 1-9.

Park, J. H., O. Kwon and S. Won (2004). LMI approach to robust $\mathcal{H}_{\infty}$ filtering for neutral delay differential systems. Applied Mathematics and Computation 150(1), 235-244.

Peaucelle, D., D. Arzelier, O. Bachelier and J. Bernussou (2000). A new robust $\mathcal{D}$-stability condition for real convex polytopic uncertainty. Systems \& Control Letters 40(1), 2130.

Ramos, D. C. W. and P. L. D. Peres (2001). A less conservative LMI condition for the robust stability of discrete-time uncertain systems. Systems \& Control Letters 43(5), 371-378.

Ramos, D. C. W. and P. L. D. Peres (2002). An LMI approach to compute robust stability domains for uncertain linear systems. IEEE Transactions on Automatic Control 47(4), 675-678.

Skorodinskii, V. I. (1990). Iterational method of construction of Lyapunov-Krasovskii functionals for linear systems with delay. Automation and Remote Control 51(9), 1205-1212.

Verriest, E. I. (1999). Robust stability and adaptive control of time-varying neutral systems. In: Proceedings of the 38th IEEE Conference on Decision and Control. Phoenix, AZ. pp. 4690-4695.

Xu, S., J. Lam, C. Yang and E. I. Verriest (2003). An LMI approach to guaranteed cost control for uncertain linear neutral delay systems. International Journal of Robust and Nonlinear Control 13(1), 35-53. 